\title{
A Rare Case Report of Colonic Perforation and Gangrene: A Sequelae of Self-inflicted Transanal Compressed Air Injury
}

\author{
Ashokan Bhaskaran, Ambikavathy Mohan
}

\begin{abstract}
In the present days of industrialization, use of compressed air jets is common. Although industries provide employment to population at large, the misuse of pneumatic equipment at work place has relatively increased. We report a case of a male patient aged 20 years who presented to us with anal bleed, pain and distension of abdomen and severe breathlessness following a self-thrusting of a vacuum blower pipe into the anal orifice for sexual stimulation. He was investigated and underwent emergency exploratory laparotomy with total colectomy of the gangrenous colon and end ileostomy. Postoperatively, the patient succumbed to a cardiac event.
\end{abstract}

Keywords: Colonic perforation, Compressed air injury, Bowel gangrene.

How to cite this article: Bhaskaran A, Mohan A. A Rare Case Report of Colonic Perforation and Gangrene: A Sequelae of Self-inflicted Transanal Compressed Air Injury. Euroasian J Hepato-Gastroenterol 2013;3(2):136-138.

Source of support: Nil

Conflict of interest: None

\section{INTRODUCTION}

Compressed air equipments are frequently used in the manufacturing industries. Misuse of such machinery for other purposes at place of work needs to be monitored. Compressed air injuries can be devastating, especially the colorectal injuries, as the high jet pressure leads to rupture/ perforation or gangrene of the bowel within a short time., ${ }^{1,2}$ Management colorectal injuries are a great challenge to the treating surgeons, as the peritoneum is usually contaminated with fecal spillage and the outcome can be fatal.

\section{CASE REPORT}

A 20 years old male patient employed in an industry was brought to the accident and emergency department by a co-employee with the history of the patient complaining of pain abdomen and distension, bleeding per rectum and breathlessness. On further interview the patient confessed to the self-thrusting of a vacuum blower pipe into the anus for sexual stimulation the previous night.

On thorough examination the patient was in severe pain, restless and appeared pale and drowsy, though he responded to the queries. He was in hypovolemic shock, tachycardia, tachypnea was present. Abdomen was grossly distended and severely tender with features of peritonitis. Subcutaneous emphysema was felt over the suprapubic and left iliac region. Liver dullness was obliterated, bowel sounds were absent. On per rectal examination the patient had severe pain with profuse fresh bleed. A rent of $1 \times 2 \mathrm{~cm}$ was felt in the rectum anteriorly. Sphincter tone was weak and patulous. We made a diagnosis of colonic perforation and tension pneumoperitoneum with fecal peritonitis with septicemia and shock, secondary to barotrauma.

After adequate resuscitation, emergency investigations were done. Hemoglobin was $8 \mathrm{gm} / \mathrm{dl}$, total count was $12,000 / \mathrm{mm}^{3}$, and creatinine level was $1.1 \mathrm{mg} / \mathrm{dl}$. X-ray of the chest and erect abdomen showed free air under diaphragm and dilated large bowel loop with multiple air fluid levels suggestive of bowel perforation (Figs 1 and 2). A complementary ultrasound reported as dilated large bowel

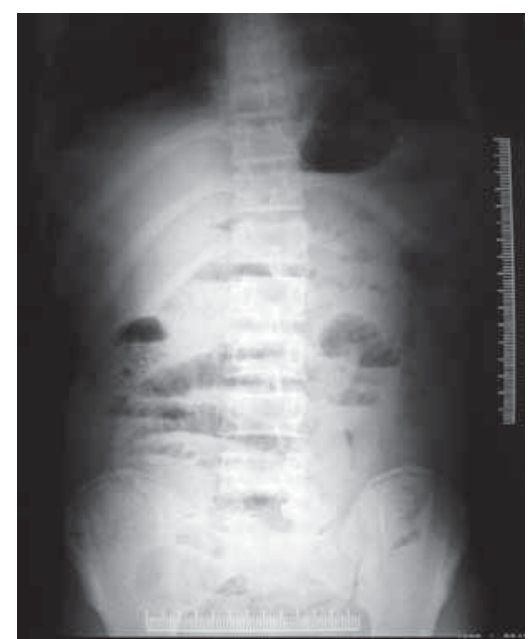

Fig. 1: Erect abdomen X-ray showing air under both the diaphragms, dilated bowel loops with multiple air fluid levels

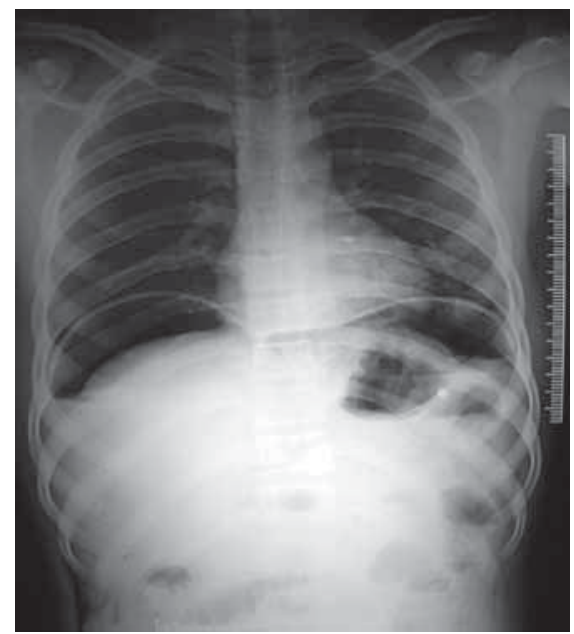

Fig. 2: Chest X-ray showing free air under both diaphragms 
loops and intraperitoneal collection, diagnostic tap was an aspirate of fecal and blood, confirming fecal peritonitis and hemoperitoneum. The abdominal tap with a large bore needle helped in releasing the pneumoperitoneum and patient was relieved of his breathlessness to some extent.

The patient was taken up for emergency exploratory laparotomy under endotracheal intubation and the operative findings were: whole of the colon from the rectosigmoid junction to the cecum was gangrenous, small bowel was viable. There were two perforations of size $1 \times 2 \mathrm{~cm}$ in the anterior wall of rectosigmoid junction and another of $2 \times$ $1 \mathrm{~cm}$ in the transverse colon along the antimesenteric border. Peritoneum was grossly contaminated with fecal spillage. There were no other solid organ injuries. We performed total colectomy with closure of distal segment and end ileostomy (Figs 3 to 7). Patient had hypotension both intraoperatively and postoperatively. He was put on a ventilator with inotropic support and nursed in the ICU. On the second postoperative day, the patient succumbed to a cardiac event despite timely intervention.

\section{DISCUSSION}

Compressed air insufflations into the anus as a sexual perversion or autoeroticism are reported in a limited number of cases. ${ }^{1}$ Compressed air apparatuses have a pressure of 50 to 150 psi. As reported by Burt in 1931, in an experimental study of the four layers of the intestine, mucosa is the strongest. Pressures of 3.99 psi can tear the muscular layer and serosa, a pressure of 4.07 ruptures the total thickness of the intestine causing perforation. ${ }^{2}$

Clinical manifestations are very accurate in diagnosing colonic perforations. Pain and distension of abdomen are early to present followed by features of peritonitis. Breathlessness as seen in our case is due to pneumoperitoneum causing restriction of diaphragmatic movements. Respiratory distress leading to tachypnea can result in respiratory alkalosis. ${ }^{3}$ Our patient had hypotension which could be due to the tension pneumoperitoneum compressing the vena cava reducing venous return and circulatory failure. Profuse loss of blood could also contribute to this.

The anatomy of the bottom and perineum is like a funnel allowing easy flow of compressed air into the anus and rectum, directed against antimesenteric border of rectosigmoid junction and is the common site to perforate. The perforations can be single or multiple. The small bowel pneumatic perforation is rare. The pathophysiology of gangrene can be explained by the fact that the over distended bowel compromises on the blood supply, followed by the thromboembolic phenomenon of the inferior mesenteric vessels leading to gangrene. ${ }^{4}$ Our patient had a rent of
$2 \times 1 \mathrm{~cm}$ in the anterior wall of rectum and a perforation $2 \times 3 \mathrm{~cm}$ in the transverse colon and small bowel was spared. Colonic injuries are unique in presentation, patients report very late to hospital as they are hesitant to reveal the history, especially in misuse of compressed air apparatus into anorectum for sexual stimulation, as it is a socially

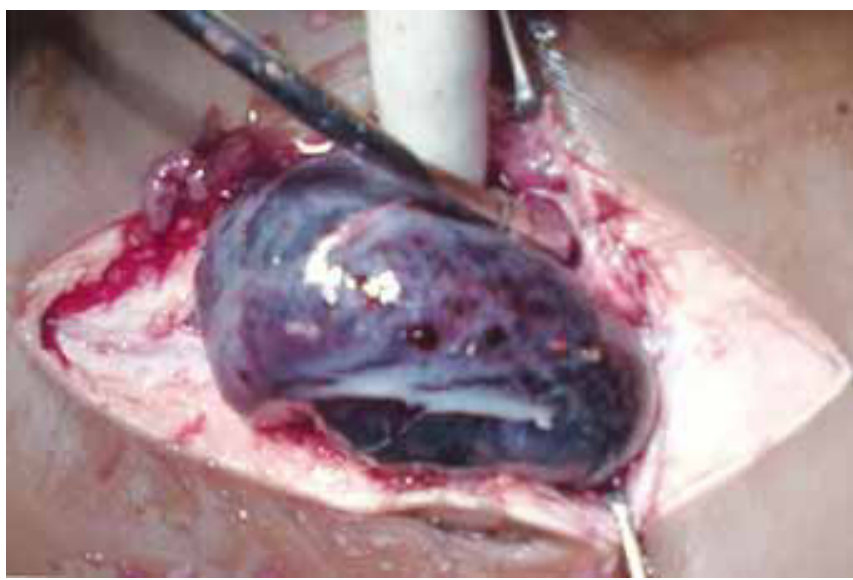

Fig. 3: Intraoperative photograph showing distended and gangrenous bowel popping out through the operative wound

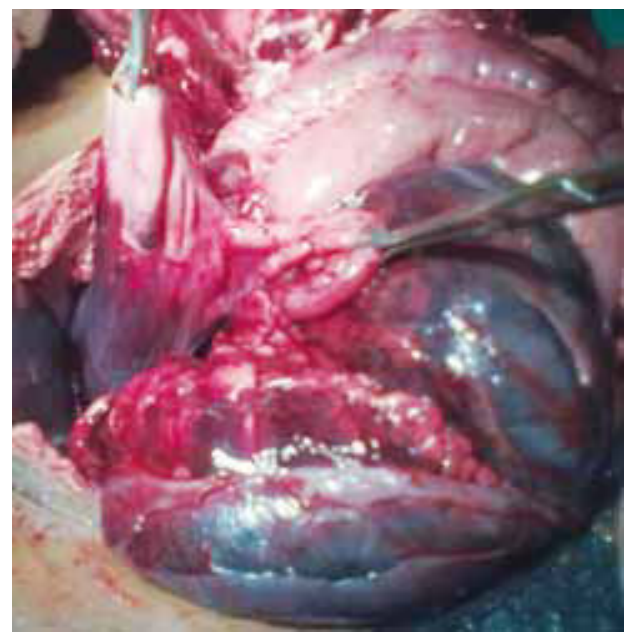

Fig. 4: Intraoperative photograph showing gangrene of large bowel extending from rectosigmoid junction to the cecum

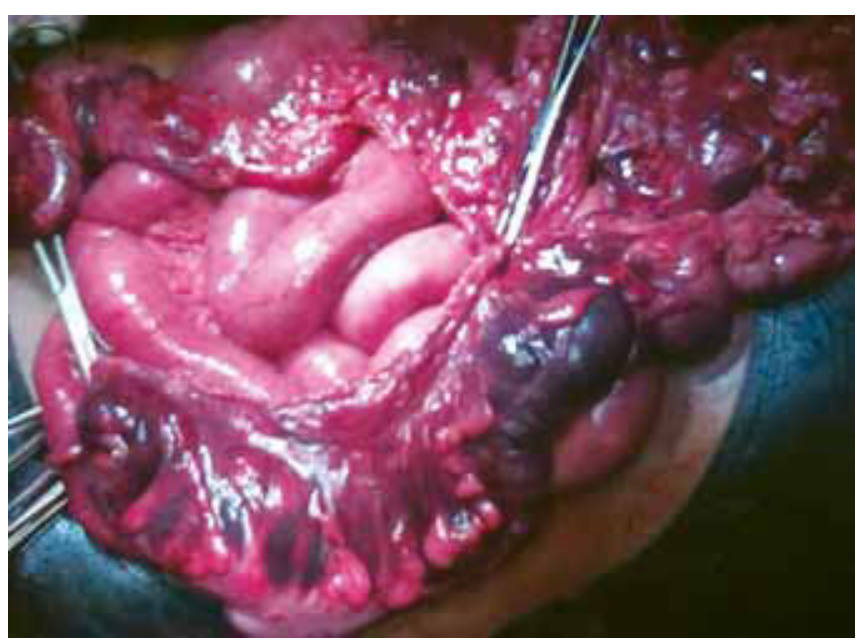

Fig. 5: Intraoperative photograph showing gangrenous large bowel with normal small bowel 


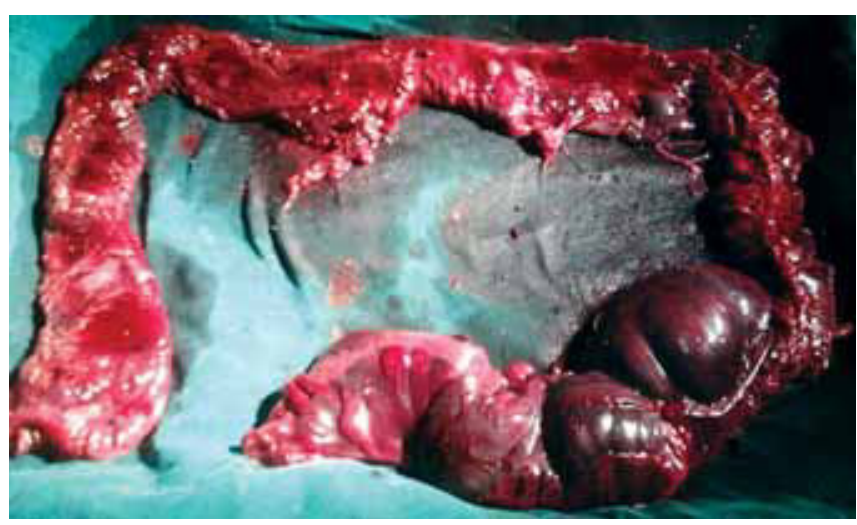

Fig. 6: Postoperative photograph of total colectomy specimen showing gangrene of the colon with perforation in transverse colon and rectosigmoid
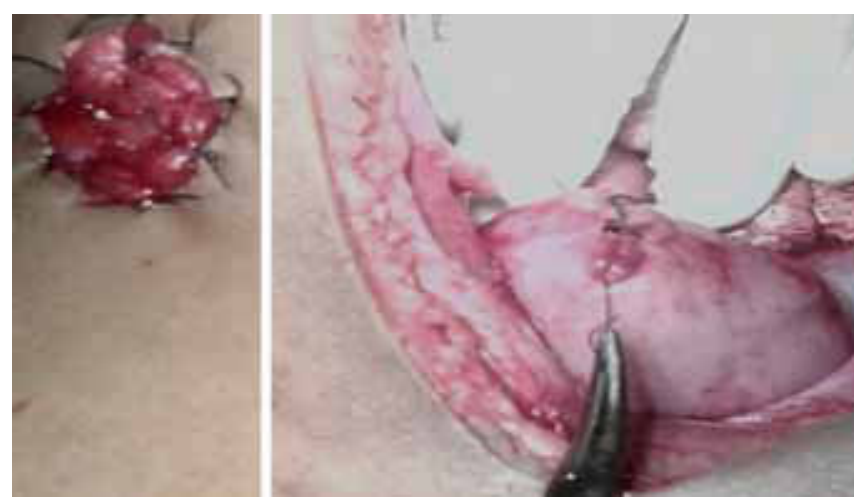

Fig. 7: Operative picture showing closure of distal bowel segment and end ileostomy

embarrassing situation. Colonic perforations can be very fatal in delayed interventions as fecal peritonitis and septicemia sets in, causing morbidity and mortality. But with recent advances in treatment, diffuse peritonitis has a mortality rate of $10 \% .{ }^{5}$ Our patient presented 24 hours after the incident and despite early intervention he succumbed postoperatively.

\section{CONCLUSION}

Colorectal injury should be suspected in patients with history of compressed air injury. Adequate resuscitation and early surgical intervention can save a patient's life. Psychiatric illness is to be considered in such cases. A proper monitoring of industrial employees is mandatory along with counseling. Education and awareness of hazards of compressed air injury can go a long way in preventing fatal injuries.

\section{REFERENCES}

1. Clarke DL, Buccimazza I, Anderson FA, Thompson SR. Colorectal foreign bodies. Colorectal Dis 2005 Jan;7(1):98-103.

2. Burt AV. Pneumatic rupture of intestinal canal with experimental data showing the mechanism of perforation and the pressure required. Arch Surg 1931 Jun;22(6):875-902.

3. Brown RK, Dwinelle JH. Rupture of colon by compressed air. Report of three cases. Ann Surg 1942 Jan;115(1):13-20.

4. Farbin S, Davisdon P, Shockley L. Perforation of the sigmoid colon by hydrostatic pressure of a public water fountain. J Emerg Med 1996 Nov-Dec;14(6):703-706.

5. Williams, Norman S.; Bulstrode, Christopher JK.; O'Connell, P Ronan. Bailey and Love's short practice of surgery. Acute peritonitis. 24th ed. London: CRC Press, Taylor \& Francis Group; 2004. 1133 p.

\section{ABOUT THE AUTHORS}

\section{Ashokan Bhaskaran}

Professor, Department of Surgery, Sri Devaraj Urs Medical College Kolar, Karnataka, India

\section{Ambikavathy Mohan (Corresponding Author)}

Assistant Professor, Department of Surgery, Sri Devaraj Urs Medical College, Kolar, Karnataka-563101, India, Phone: 9980337428 e-mail: ambikaashri67@live.com 